\title{
Efficacy, effectiveness, and safety of integrase inhibitors in the treatment of HIV/AIDS in patients with tuberculosis: A systematic review and meta-analysis
}

\author{
Natália Resende $^{1}$, Lais Pantuzza ${ }^{1}$, Wânia Carvalho ${ }^{1}$, Silvana Miranda ${ }^{1}$, Isabella Zuppo ${ }^{1}$, \\ and Adriano Reis ${ }^{1}$ \\ ${ }^{1}$ Universidade Federal de Minas Gerais
}

August 26, 2021

\begin{abstract}
Aims: To evaluate the efficacy, effectiveness, and safety of integrase inhibitors in the treatment of HIV/AIDS in patients coinfected with tuberculosis (TB). Methods: Clinical trials or observational studies were included. The searches were performed in the MEDLINE, EMBASE, LILACS, COCHRANE, Web of Science, Scopus, and CINAHL databases using the terms "HIV", "AIDS", "tuberculosis", "raltegravir potassium", "dolutegravir", "elvitegravir", "bictegravir", "integrase inhibitor", and their respective synonyms. The methodological quality of the studies was independently assessed using the Cochrane risk of bias and Newcastle Ottawa scales. Results: Reports from three randomised clinical trials and a historical cohort were included. Patients coinfected with TB and HIV/AIDS showed a good response to TB treatment, which was above $85 \%$ in all arms of the evaluated studies. As a primary outcome, the HIV viral load suppression rates at week 48 were greater than $60 \%$ in all arms. The therapies evaluated in patients coinfected with TB and HIV/AIDS were also proven to be safe. However, there was no statistically significant difference in the efficacy outcomes between the efavirenz and integrase inhibitor arms, and regarding safety outcomes, there were few events compared with the total. Furthermore, the certainty of the evidence of the outcomes assessed was low, indicating that future research is likely to have an important impact on this estimate. Conclusion: Integrase inhibitors are effective and well tolerated, being an alternative to efavirenz in clinical protocols. However, more studies with high quality evidence are needed on the use of this treatment in health systems.
\end{abstract}

\section{Hosted file}

Manuscript.docx available at https://authorea.com/users/431813/articles/535296-efficacyeffectiveness-and-safety-of-integrase-inhibitors-in-the-treatment-of-hiv-aids-inpatients-with-tuberculosis-a-systematic-review-and-meta-analysis 